\title{
MAGIC observations of the nearby short GRB 160821B
}

\author{
Koji Noda, ${ }^{a, *}$ Lara Nava, ${ }^{b, c}$ Susumu Inoue, ${ }^{d, e}$ Satoshi Fukami, ${ }^{a}$ Michele Palatiello,,${ }^{f, c}$ \\ Alessio Berti $^{g}$ and Francesco Longo ${ }^{h, c}$ on behalf of the MAGIC and Fermi/LAT \\ Collaboration ${ }^{\dagger}$ \\ ${ }^{a}$ Institute for Cosmic Ray Research, The University of Tokyo, Kashiwanoha 5-1-5, Kashiwa, Japan \\ ${ }^{b}$ INAF - Osservatorio Astronomico di Brera, via Emilio Bianchi 46, Merate, Italy \\ ${ }^{c}$ INFN, Sezione di Trieste, via Valerio 2, Trieste, Italy \\ ${ }^{d}$ Bunkyo University, Minami-Ogishima 3337, Koshigaya, Japan \\ ${ }^{e}$ RIKEN, Hirosawa 2-1, Wako, Japan \\ ${ }^{f}$ University of Udine, Via Palladio 8, Udine, Italy \\ ${ }^{g}$ Max Planck Institute for Physics, Föhringer Ring 6, Munich, Germany \\ ${ }^{h}$ University of Trieste, Department of Physics, Via Valerio 2, Trieste, Italy \\ E-mail: nodak@icrr.u-tokyo.ac.jp
}

Gamma-ray bursts (GRBs), the most luminous explosions in the universe, have at least two types known. One of them, short GRBs, have been thought to originate from binary neutron star (BNS) mergers. The discovery of GW170817 together with a GRB was the first and only direct proof of the hypothesis, and thus the properties of the short GRBs are poorly known yet. Aiming to clarify the underlying physical mechanisms of the short GRBs, we analyzed GRB 160821B, one of the nearest short GRBs known at $\mathrm{z}=0.162$, observed with the MAGIC telescopes. A hint of a gamma-ray signal is found above $0.5 \mathrm{TeV}$ at a significance of $>3$ sigma during observations from 24 seconds until 4 hours after the burst, as presented in the past. Recently, multi-wavelength data of its afterglow emission revealed a well-sampled kilonova component from a BNS merger, and the importance of GRB 160821B increased concerning GRB-GW studies. Accordingly, we investigated GRB afterglow models again, using the revised multi-wavelength data. We found that the straightforward interpretation with one-zone synchrotron self-Compton model from the external forward shock is in tension with the observed $\mathrm{TeV}$ flux, contradicting the suggestion reported previously. In this contribution we discuss the implication from the $\mathrm{TeV}$ observation, including alternative scenarios where the $\mathrm{TeV}$ emission can be enhanced. We also give a brief outlook of future GeV-TeV observations of short GRBs with imaging atmospheric Cherenkov telescopes, which could shed more light on the GRB-BNS merger relation.

$37^{\text {th }}$ International Cosmic Ray Conference (ICRC 2021)

July 12 th - 23rd, 2021

Online - Berlin, Germany

\footnotetext{
${ }^{*}$ Presenter

${ }^{\dagger}$ a complete list of the MAGIC Collaboration authors can be found at the end of the proceedings
} 


\section{Introduction}

Gamma-ray bursts (GRBs) are known as the most luminous explosions in the universe, which emit photons of energies ranging from $\mathrm{keV}$ to $\mathrm{MeV}$ as a short pulse, called the prompt emission. It is followed by a slower afterglow emission that exhibits a wide range of its duration from minutes to months, in the wavelengths ranging from radio to high-energy gamma-rays. The duration and spectrum of the prompt emission have a bimodal distribution, and it is believed as an indication of two different classes, named Long and Short GRBs. Long GRBs are widely believed to be produced by the core collapse of massive stars [1], with tens of observational confirmations of an accompanying supernova explosion. On the other hand, the origin of Short GRBs has been less clear, with an indication for mergers of binary neutron stars by studying their circumstances [2]. The strong but singular support for the indication was the discovery of GW170817 coincident with GRB 170817A [3], followed by an optical-infrared emission called kilonova [4].

GRBs with $T_{90}{ }^{1}$ less than 2 seconds are regarded as Short GRBs, while the others as Long GRBs. However, it is not simple to classify all the observed GRBs into the two types. The bimodal distribution of the prompt emission is known to have an intrinsic overlap, due to GRBs with an ambiguous duration and/or hardness of their spectrum ${ }^{2}$. Also, there is an observational bias; the prompt emission is often just a single fast-rising peak with an exponential decay, and thus, if the GRB is far and dim, its duration will appear shorter than in the case where it would exist closer. It is known that the typical circumburst environments such as the density of ambient material are also different between the two types. The afterglow emission depends on such physics parameters so it can help with the classification, but it is often not sufficient. In the end, a GRB with marginal conditions can be clearly classified only with a confirmation of a supernova or a kilonova by optical-infrared observations that are carried out typically days/weeks after the burst.

Short GRBs are relatively dimmer than Long GRBs, so their optical observations are more difficult in general. On top of that, the expected kilonova emission is intrinsically dimmer than the supernova emission. Thus, there are only a few Short GRBs known to be accompanied by a possible kilonova emission, such as GRB 130603B $\left(T_{90}=0.18 \mathrm{~s}, z=0.356\right)$ [8]. There are also only a few GRBs with a short (intrinsic) duration $T_{90}$ but with a supernova emission, such as GRBs $040924\left(T_{90}=2.4 \mathrm{~s}, z=0.86\right)$ [5] and 200826A ( $\left.T_{90} \sim=0.5 \mathrm{~s}, z=0.75\right)$ [6, 7]. Most of Short GRBs are lacking a kilonova/supernova confirmation, and consequently their properties are much less understood than Long GRBs. More discoveries of coincident multi-wavelength / messenger events are required to understand their properties.

GRB 160821B $\left(T_{90}=0.48 \mathrm{~s}, z=0.162\right)$ is one of few Short GRBs with a confirmed kilonova emission. Its multi-wavelength observations were carried out including radio, optical, X-ray, $\mathrm{GeV}$ photons, and very-high-energy (VHE, $>100 \mathrm{GeV}$ ) gamma-rays using imaging atmospheric Cherenkov telescopes. MAGIC telescopes found a hint of a very-high-energy gamma-ray emission, reported in the conferences $[12,13]$. However, the kilonova emission was just constrained in the first reports of the optical observations [9]. More extensive investigations only after the discovery of GW170817 revealed the second most sampled kilonova emission next to GRB 170817A, published in 2019 [10, 11]. In the 36th ICRC in 2019 we showed a multi-wavelength modeling to explain the

\footnotetext{
${ }^{1}$ The duration of GRBs is commonly measured by $T_{90}$, the time interval containing $90 \%$ of the prompt photons.
}

${ }^{2}$ There are also interpretations to assume a third class. 
VHE emission but without a complete interpretation of all the currently available multi-wavelength information [14]. Moreover, the discovery of TeV gamma-ray emissions from Long GRBs in 2019 [15-17] made the hint of detection more plausible. A revision of the interpretation of the possible VHE emission from Short GRB 160821B became more important than before, which is the subject of this contribution.

A significant fraction of this contribution has been published in [18].

\section{Observations}

GRB 160821B triggered Swift-BAT at 22:29:13 UT on 2016 August $21\left(T_{0}\right)$. The $T_{90}$ duration reported by Swift-BAT was 0.48 s [19], while that by Fermi-GBM was about $1 \mathrm{~s}$ [20]. Both indicate that the GRB is fairly inside the Short GRB regime. A host galaxy was identified with redshift of $z=0.162$ [21], which revealed that this GRB is one of the nearest Short GRBs. The isotropic energy is estimated to be $E_{\text {iso }} \sim 1.2 \times 10^{49} \mathrm{erg}$, which is relatively low for a Short GRB but not extremely. The follow-up observations with multi-wavelength instruments were carried out, and they are briefly introduced below. See the paper [18] for further details.

MAGIC started to observe GRB 160821B at 22:29:37 UT, $24 \mathrm{~s}$ after $T_{0}$, and continued until $4 \mathrm{hr}$ after $T_{0}$. The zenith angle of the source increased from $34 \mathrm{deg}$ to $55 \mathrm{deg}$, and the night sky background light increased as moon rose. As a result the energy threshold of the obtained data should be increasing with time. However, the data taken in the first $\sim 1.7 \mathrm{hr}$ were strongly affected by clouds, while the remaining $\sim 2.2 \mathrm{hr}$ were taken under better weather conditions. Consequently, the energy threshold is as high as $0.5 \mathrm{TeV}$ for the whole data sample of $4 \mathrm{hr}$.

At the time of the trigger by Fermi-GBM, the burst was near the border of the standard FoV of the Fermi-LAT detector $(60 \mathrm{deg})$. A non-standard FoV limit of $\sim 70$ deg allows us to analyze the data of GRB 160821B from $T_{0}$ to $T_{0}+2315 \mathrm{~s}$. The source entered the FoV again later, from $T_{0}+$ $5285 \mathrm{~s}$ to $T_{0}+8050 \mathrm{~s}$.

The optical afterglow was first reported by NOT [22], and confirmed by WHT [21], GTC [23], and HST, which reported the redshift of $z=0.162$ [9]. Observations confirmed that the source was fading in the optical band, as is the case for most of GRBs, with a magnitude $r=22.6$ mag at 0.95 hr after $T_{0}$. The GRB is located in the outskirts of a host spiral galaxy. In the radio band, VLA detected a fading source consistent with the afterglow from the GRB [24].

\section{Results}

MAGIC detected a hint of the gamma-ray emission above $\sim 0.8 \mathrm{TeV}$ with a significance of 3.1 sigma (pre-trial), which leads to 2.9 sigma after a correction for the cuts tried in the analysis (Fig.1). The right panel of Fig. 1 shows the sky map of the excess significance (pre-trial over the FoV), which is consistent with the excess signals derived from the so-called theta squared plot (the left panel), where the events are plotted with respect to the angular distance from the source squared. A slight discrepancy from the source location is seen in the sky map, which can be well attributed to the statistical fluctuation [18].

In order to estimate the flux inferred from the hint of the detection, we divided the data into two parts differentiated by the weather conditions (see Sec.2). Then, we further subdivided the first 

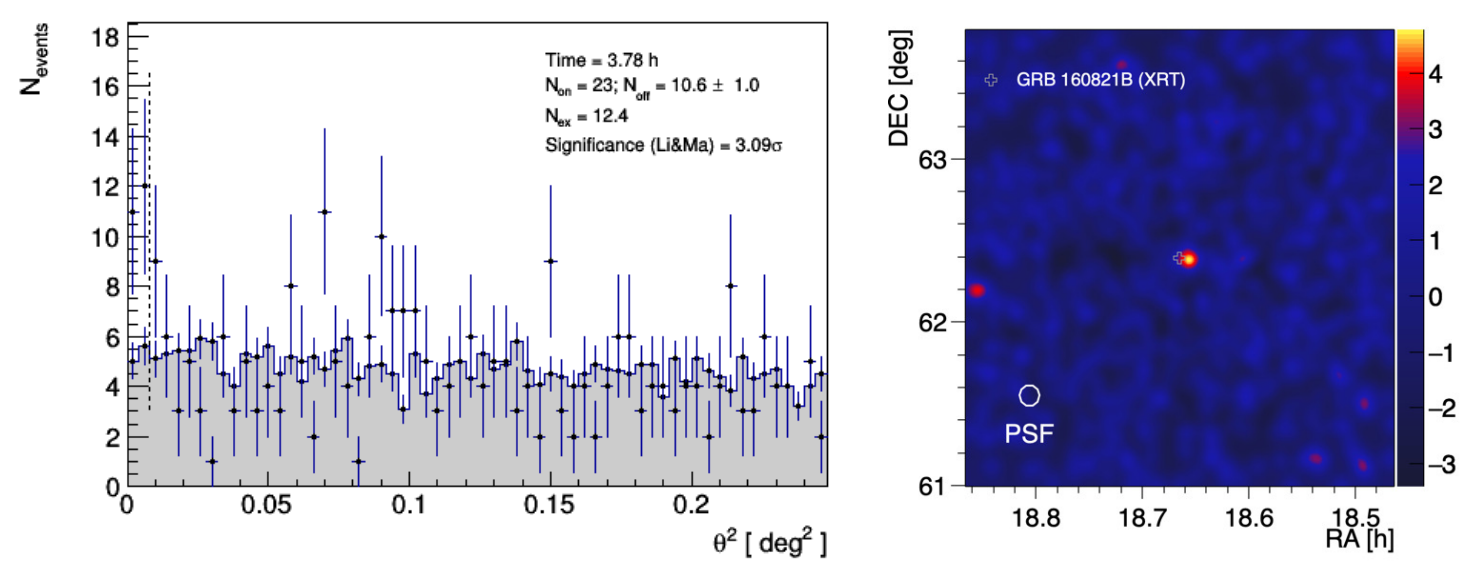

Figure 1: The hint of the detection by MAGIC [18]. Left: so-called theta square plot, where the events are plotted with respect to the angular distance from the source squared, showing a hint of the detection with 3.1 sigma. Right: the corresponding skymap with pre-trial significance of 4.7 sigma.

part into two bins to well represent the trend in the logarithmic scale in terms of time since $T_{0}$. Out of the three bins in total, the first 2 bins $\left(t-T_{0}=24-1216 \mathrm{~s}\right.$, and $\left.1258-6098 \mathrm{~s}\right)$ provided us only with an upper limit point. On the other hand, we could derive an indicative flux point for the last bin (6134 - $14130 \mathrm{~s})$, together with an upper limit. All the flux / upper limit points are derived with an energy threshold of $0.5 \mathrm{TeV}$, with assuming the power-law index of -2 (red points in Fig.2). The flux value was translated into a spectrum by fixing an energy range to $0.5-5 \mathrm{TeV}$, as seen in the thin red box in Fig.3.

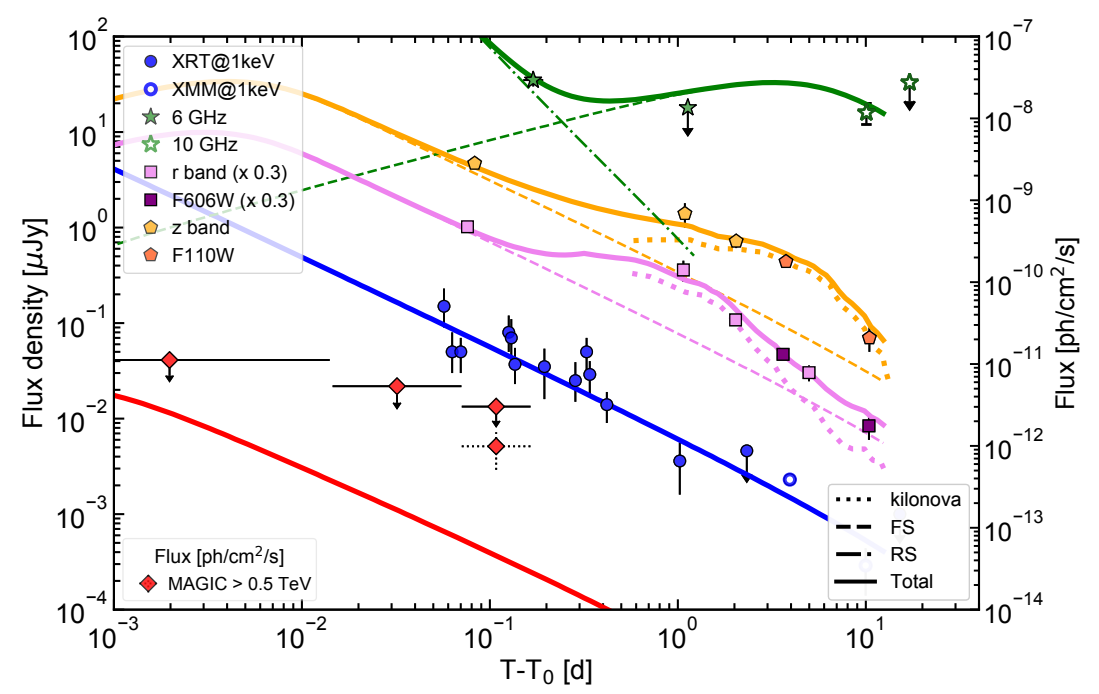

Figure 2: Multiwavelength light curve with the model curves [18]. See texts for details.

No emission was detected by Fermi-LAT in the energy range $0.3-3 \mathrm{GeV}$. Since the upper limits are less constraining, they are not drawn in the following figures (See Fig.1 of [18]). The Fermi-LAT 


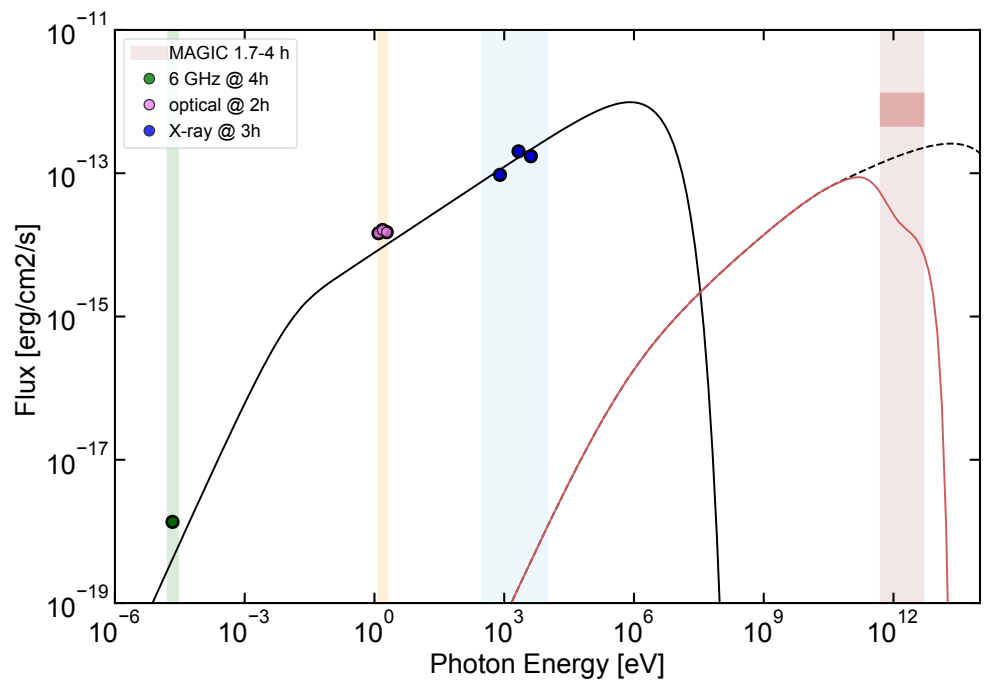

Figure 3: Spectral energy distribution with the model curves [18]. Dashed line is the source intrinsic flux after the EBL attenuation is corrected for. See texts for more details.

observations are taken into account in the following discussions, even though not explicitly written.

\section{Discussions}

The modeling has two steps; the first step is the modeling of the emission from radio to X-ray, then the TeV radiation is modeled based on the first step. The first step for GRB 160821B is not simple, as it has more components than typical GRBs have. The afterglow emission common for Short and Long GRBs is synchrotron radiation from electrons accelerated in external forward shocks, triggered by interactions between the relativistic jet and the ambient medium. It is sometimes accompanied with a reverse shock propagating into the jet ejecta, which is the case for this GRB. On top of this afterglow emission with a reverse shock, it has a so-called extended emission as Short GRBs often have, which means an X-ray emission extended in time for tens to hundreds of seconds followed by a steep decay. It is thought to be related to long-lasting activity of the central engine. Finally, the kilonova emission, which is powered by synthesized r-process elements ejected in neutron star mergers, can occur on timescale of days [4] but is rarely observed, as mentioned in Sec.1. The lines in Fig.2 shows our model to explain the radio to X-ray observations. Other than the extended emission that occurs earlier than the plotted range, the three components, forward and reverse shocks and the kilonova emissions, are consistently described by the single model.

The first step suggests that the optical data at $2 \mathrm{hr}$ are dominated by the forward shock component before the kilonova emission emerged, while the radio data at $4 \mathrm{hr}$ are dominated by the reverse shock component. We modeled the broadband emission at $t \sim t_{0}+3 \mathrm{hr}$, including the MAGIC observation, as synchrotron emission from the external forward shock, considering the simplest case of impulsive energy injection. The modeling is shown in Fig.3, which is performed with a numerical code [16] that self-consistently solves the evolution of the electron distribution, accounting for continuous 
electron injection with a power-law energy distribution, synchrotron, synchrotron-self-Compton (SSC) \& adiabatic losses, synchrotron self-absorption, and $\gamma \gamma$ pair production. The estimated $\mathrm{TeV}$ flux is shown as red solid line both in Figs. 2 and 3, to be compared with the flux inferred by MAGIC. The adopted one-zone synchrotron self-Compton model from the external forward shock is one of the most straightforward interpretations, which however predicts more than one order of magnitude lower flux than the flux inferred from the observation. See [18] for further details of the parameters. We conclude that the simplest model is in tension with the observed TeV flux.

There are possibilities of other interpretations. First considered is the proton synchrotron emission [25]. From a simple calculation [18] the inferred maximum photon energy and flux require too large material density and/or kinetic energy. Thus, the interpretation is strongly disfavored, similarly to other interpretations like photohadronic cascade [26]. One potentially plausible interpretation would be the external Compton emission [27]. There are a few possibilities of the target soft photons such as the X-ray extended emission and kilonova, as well as the thermal X-rays in the forward shock, all of which are observed in GRB 160821B. Finally, the GRB clearly showed the reverse shock component, which would allow a TeV component still in the context of SSC [28], possibly with continuous energy injection [29].

\section{Summary and Outlook}

The classification of GRBs only with their duration is prone to ambiguity, in particular around $T_{0} \sim 2 \mathrm{~s}$. The kilonova emission observed in optical band is thought to be a confirmation of Short GRB type, which is however difficult to detect due to its dimness.

GRB 160821B is one of the nearest Short GRBs, observed by multi-wavelength instruments from radio even to (very-)high-energy gamma-ray instruments such as MAGIC telescopes and Fermi-LAT. The multi-wavelength observations revealed the X-ray extended emission, forward and reverse shock afterglow, and the kilonova emission. On top of that, MAGIC has reported a hint of VHE gamma-ray emission, which made this GRB more intriguing.

A new modeling including the most recent multi-wavelength data sets is reported in this contribution. The modeling showed that the simplest interpretation with the SSC model does not well reproduce the observations of the VHE gamma-ray emission. Possible alternative models such as the proton synchrotron are discussed.

This observation is an important step for the multi-messenger observation of GRBs including GW detectors. NS-NS mergers are one of the key targets of the GW detectors, such as LIGOVirgo-KAGRA, and their operation started recently. For the coming years, not only the currently operational Cherenkov telescopes (such as MAGIC, H.E.S.S., VERITAS) but also the next project, Cherenkov Telescope Array, will be essential to study the GRB-GW events, and understand the nature of Short GRBs.

\section{Acknowledgments}

We acknowledge the support from the agencies and organizations listed here:

https://magic.mpp.mpg.de/acknowledgments_ICRC2021 
The Fermi-LAT Collaboration acknowledges support for LAT development, operation and data analysis from NASA and DOE (United States), CEA/Irfu and IN2P3/CNRS (France), ASI and INFN (Italy), MEXT, KEK, and JAXA (Japan), and the K.A. Wallenberg Foundation, the Swedish Research Council and the National Space Board (Sweden). Science analysis support in the operations phase from INAF (Italy) and CNES (France) is also gratefully acknowledged. This work performed in part under DOE Contract DE-AC02-76SF00515.

\section{References}

[1] S. E. Woosley and J. S. Bloom, Annual Review of Astronomy and Astrophysics, 44 (2006) 507.

[2] E. Berger, Annual Review of Astronomy and Astrophysics, 52 (2014) 43.

[3] B. P. Abbott, R. Abbott, T. D. Abbott, et al., ApJL, 848 (2017) L12.

[4] B. D. Metzger Living Reviews in Relativity, 23 (2020) 1.

[5] A. M. Soderberg, S. R. Kulkarni, P. A. Price, et al., ApJ, 636 (2006) 391.

[6] A. Rossi et al., (2021) [astro-ph/2105.03829].

[7] T. Ahumada et al., (2021) [astro-ph/2105.05067].

[8] N. R. Tanvir, A. J. Levan, A. S. Fruchter, et al., Nature, 500 (2013) 547.

[9] E. Troja, N. Tanvir, S. B. Cenko, et al., GCN Circ. (2016) 20222.

[10] G. P. Lamb, N. R. Tanvir, A. J. Levan, et al. ApJ, 883 (2019) 48.

[11] E. Troja, A. J. Castro-Tirado, J. Becerra González, et al. MNRAS, 489 (2019) 2104.

[12] K. Noda, et al. (for MAGIC Collaboration), The 29th International Texas Symposium (Cape Town, South Africa) (2017).

[13] S. Inoue, et al. (for MAGIC Collaboration), The 35th ICRC (Busan, Korea) (2017).

[14] S. Inoue, et al. (for MAGIC Collaboration), Proc. of 36th ICRC (Madison, USA) (2019) 703.

[15] MAGIC Collaboration, Nature, 575 (2019) 455.

[16] V. A. Acciari, et al., Nature, 575 (2019) 459.

[17] H. Abdalla, R. Adam, F. Aharonian, et al., Nature, 575 (2019) 464.

[18] V. A. Acciari, et al., ApJ, 908 (2021) 90.

[19] M. H. Siegel, S. D. Barthelmy, D. N. Burrows, et al., GCN Circ., (2016) 19833.

[20] M. Stanbro \& C. Meegan, GCN Circ., (2016) 19843.

[21] A. J. Levan, K. Wiersema, N. R. Tanvir, et al., GCN Circ., (2016) 19846. 
[22] D. Xu, D. Malesani, A. de Ugarte Postigo, et al., GCN Circ., (2016) 19834.

[23] S. Jeong, I. H. Park, Y. Hu, et al., GCN Circ., (2016) 19847.

[24] W. Fong, K.D. Alexander, \& T. Laskar, GCN Circ., (2016) 19854.

[25] M. Vietri, PRL, 78 (1997) 4328.

[26] M, Böttcher, \& C. D. Dermer, ApJL, 499 (1998) L131.

[27] K. Murase, K. Toma, R. Yamazaki, \& P. Mészáros, ApJ, 732 (2011) 77.

[28] X. Y. Wang, Z. G. Dai, \& T. Lu, ApJ, 556 (2001) 1010.

[29] P. Veres, \& P. Mészáros, ApJ, 787 (2014) 168.

\section{The MAGIC Collaboration}

V. A. Acciari ${ }^{1}$, S. Ansoldi ${ }^{2,41}$, L. A. Antonelli ${ }^{3}$, A. Arbet Engels ${ }^{4}$, M. Artero ${ }^{5}$, K. Asano ${ }^{6}$, D. Baack ${ }^{7}$, A. Babié ${ }^{8}$, A. Baquero ${ }^{9}$, U. Barres de Almeida ${ }^{10}$, J. A. Barrio ${ }^{9}$, I. Batković ${ }^{11}$, J. Becerra González ${ }^{1}$, W. Bednarek ${ }^{12}$, L. Bellizzi ${ }^{13}$, E. Bernardini ${ }^{14}$, M. Bernardos ${ }^{11}$, A. Berti ${ }^{15}$, J. Besenrieder ${ }^{15}$, W. Bhattacharyya ${ }^{14}$, C. Bigongiari ${ }^{3}$, A. Biland ${ }^{4}$, O. Blanch ${ }^{5}$, H. Bökenkamp ${ }^{7}$, G. Bonnoli ${ }^{16}$, Ž. Bošnjak ${ }^{8}$, G. Busetto ${ }^{11}$, R. Carosi ${ }^{17}$, G. Ceribella ${ }^{15}$, M. Cerruti ${ }^{18}$, Y. Chai ${ }^{15}$, A. Chilingarian ${ }^{19}$, S. Cikota $^{8}$, S. M. Colak ${ }^{5}$, E. Colombo ${ }^{1}$, J. L. Contreras ${ }^{9}$, J. Cortina ${ }^{20}$, S. Covino ${ }^{3}$, G. D’Amico ${ }^{15,42}$, V. D’Elia ${ }^{3}$, P. Da Vela ${ }^{17,43}$, F. Dazzi ${ }^{3}$, A. De Angelis ${ }^{11}$, B. De Lotto ${ }^{2}$, M. Delfino ${ }^{5,44}$, J. Delgado ${ }^{5,44}$, C. Delgado Mendez $^{20}$, D. Depaoli ${ }^{21}$, F. Di Pierro ${ }^{21}$, L. Di Venere ${ }^{22}$, E. Do Souto Espiñeira ${ }^{5}$, D. Dominis Prester $^{23}$, A. Donini ${ }^{2}$, D. Dorner ${ }^{24}$, M. Doro ${ }^{11}$, D. Elsaesser ${ }^{7}$, V. Fallah Ramazani ${ }^{25,45}$, A. Fattorini ${ }^{7}$, M. V. Fonseca ${ }^{9}$, L. Font ${ }^{26}$, C. Fruck ${ }^{15}$, S. Fukami ${ }^{6}$, Y. Fukazawa ${ }^{27}$, R. J. García López ${ }^{1}$, M. Garczarczyk ${ }^{14}$, S. Gasparyan ${ }^{28}$, M. Gaug ${ }^{26}$, N. Giglietto ${ }^{22}$, F. Giordano ${ }^{22}$, P. Gliwny ${ }^{12}$, N. Godinović ${ }^{29}$, J. G. Green ${ }^{3}$, D. Green ${ }^{15}$, D. Hadasch ${ }^{6}$, A. Hahn ${ }^{15}$, L. Heckmann ${ }^{15}$, J. Herrera ${ }^{1}$, J. Hoang ${ }^{9,46}$, D. Hrupec ${ }^{30}$, M. Hütten ${ }^{15}$, T. Inada ${ }^{6}$, K. Ishio ${ }^{12}$, Y. Iwamura ${ }^{6}$, I. Jiménez Martínez ${ }^{20}$, J. Jormanainen ${ }^{25}$, L. Jouvin ${ }^{5}$, M. Karjalainen ${ }^{1}$, D. Kerszberg ${ }^{5}$, Y. Kobayashi' ${ }^{6}$, H. Kubo ${ }^{31}$, J. Kushida ${ }^{32}$, A. Lamastra ${ }^{3}$, D. Lelas ${ }^{29}$, F. Leone ${ }^{3}$, E. Lindfors ${ }^{25}$, L. Linhoff ${ }^{7}$, S. Lombardi ${ }^{3}$, F. Longo ${ }^{2,47}$, R. López-Coto ${ }^{11}$, M. López-Moya ${ }^{9}$, A. López-Oramas ${ }^{1}$, S. Loporchio ${ }^{22}$, B. Machado de Oliveira Fraga ${ }^{10}$, C. Maggio ${ }^{26}$, P. Majumdar ${ }^{33}$, M. Makariev ${ }^{34}$, M. Mallamaci ${ }^{11}$, G. Maneva ${ }^{34}$, M. Manganaro ${ }^{23}$, K. Mannheim ${ }^{24}$, L. Maraschi ${ }^{3}$, M. Mariotti ${ }^{11}$, M. Martínez ${ }^{5}$, D. Mazin ${ }^{6,15}$, S. Menchiari ${ }^{13}$, S. Mender ${ }^{7}$, S. Mićanović2 ${ }^{23}$, D. Miceli ${ }^{2,49}$, T. Miener ${ }^{9}$, J. M. Miranda ${ }^{13}$, R. Mirzoyan ${ }^{15}$, E. Molina ${ }^{18}$, A. Moralejo ${ }^{5}$, D. Morcuende ${ }^{9}$, V. Moreno ${ }^{26}$, E. Moretti ${ }^{5}$, T. Nakamori ${ }^{35}$, L. Nava ${ }^{3}$,

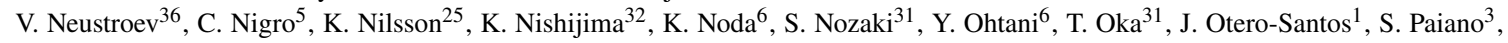
M. Palatiello ${ }^{2}$, D. Paneque ${ }^{15}$, R. Paoletti ${ }^{13}$, J. M. Paredes ${ }^{18}$, L. Pavletić ${ }^{23}$, P. Peñil ${ }^{9}$, M. Persic ${ }^{2,50}$, M. Pihet ${ }^{15}$, P. G. Prada Moroni ${ }^{17}$, E. Prandini ${ }^{11}$, C. Priyadarshi ${ }^{5}$, I. Puljak ${ }^{29}$, W. Rhode ${ }^{7}$, M. Ribó ${ }^{18}$, J. Rico ${ }^{5}$, C. Righi ${ }^{3}$, A. Rugliancich ${ }^{17}$, N. Sahakyan ${ }^{28}$, T. Saito ${ }^{6}$, S. Sakurai ${ }^{6}$, K. Satalecka ${ }^{14}$, F. G. Saturni ${ }^{3}$, B. Schleicher ${ }^{24}$, K. Schmidt $^{7}$, T. Schweizer ${ }^{15}$, J. Sitarek ${ }^{12}$, I. Šnidarić ${ }^{37}$, D. Sobczynska ${ }^{12}$,

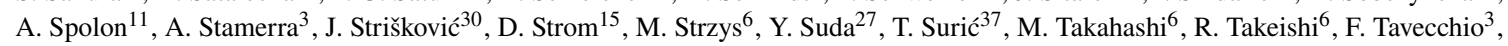
P. Temnikov ${ }^{34}$, T. Terzić ${ }^{23}$, M. Teshima ${ }^{15,6}$, L. Tosti $^{38}$, S. Truzzi ${ }^{13}$, A. Tutone ${ }^{3}$, S. Ubach ${ }^{26}$, J. van Scherpenberg ${ }^{15}$, G. Vanzo ${ }^{1}$, M. Vazquez Acosta ${ }^{1}$, S. Ventura ${ }^{13}$, V. Verguilov ${ }^{34}$, C. F. Vigorito ${ }^{21}$, V. Vitale ${ }^{39}$, I. Vovk $^{6}$, M. Will ${ }^{15}$, C. Wunderlich ${ }^{13}$, T. Yamamoto $^{40}$, and D. Zaric ${ }^{29}$

${ }^{1}$ Instituto de Astrofísica de Canarias and Dpto. de Astrofísica, Universidad de La Laguna, E-38200, La Laguna, Tenerife, Spain ${ }^{2}$
Università di Udine and INFN Trieste, I-33100 Udine, Italy ${ }^{3}$ National Institute for Astrophysics (INAF), I-00136 Rome, Italy ${ }^{4}$ ETH
Zürich, CH-8093 Zürich, Switzerland ${ }^{5}$ Institut de Física d'Altes Energies (IFAE), The Barcelona Institute of Science and Technology
(BIST), E-08193 Bellaterra (Barcelona), Spain 6 Japanese MAGIC Group: Institute for Cosmic Ray Research (ICRR), The University
of Tokyo, Kashiwa, 277-8582 Chiba, Japan ${ }^{7}$ Technische Universität Dortmund, D-44221 Dortmund, Germany ${ }^{8}$ Croatian MAGIC
Group: University of Zagreb, Faculty of Electrical Engineering and Computing (FER), 10000 Zagreb, Croatia ${ }^{9}$ IPARCOS Institute and
EMFTEL Department, Universidad Complutense de Madrid, E-28040 Madrid, Spain ${ }^{10}$ Centro Brasileiro de Pesquisas Físicas (CBPF),
$22290-180$ URCA, Rio de Janeiro (RJ), Brazil ${ }^{11}$ Università di Padova and INFN, I-35131 Padova, Italy ${ }^{12}$ University of Lodz, Faculty of
Physics and Applied Informatics, Department of Astrophysics, $90-236$ Lodz, Poland ${ }^{13}$ Università di Siena and INFN Pisa, I-53100 Siena,
Italy ${ }^{14}$ Deutsches Elektronen-Synchrotron (DESY), D-15738 Zeuthen, Germany ${ }^{15}$ Max-Planck-Institut für Physik, D-80805 München,
Germany ${ }^{16}$ Instituto de Astrofísica de Andalucía-CSIC, Glorieta de la Astronomía s/n, 18008 , Granada, Spain ${ }^{17}$ Università di Pisa and
INFN Pisa, I-56126 Pisa, Italy ${ }^{18}$ Universitat de Barcelona, ICCUB, IEEC-UB, E-08028 Barcelona, Spain ${ }^{19}$ Armenian MAGIC Group:
A. Alikhanyan National Science Laboratory, 0036 Yerevan, Armenia ${ }^{20}$ Centro de Investigaciones Energéticas, Medioambientales y
Tecnológicas, E-28040 Madrid, Spain ${ }^{21}$ INFN MAGIC Group: INFN Sezione di Torino and Università degli Studi di Torino, I-10125
Torino, Italy ${ }^{22}$ INFN MAGIC Group: INFN Sezione di Bari and Dipartimento Interateneo di Fisica dell'Università e del Politecnico di 
Bari, I-70125 Bari, Italy ${ }^{23}$ Croatian MAGIC Group: University of Rijeka, Department of Physics, 51000 Rijeka, Croatia ${ }^{24}$ Universität Würzburg, D-97074 Würzburg, Germany ${ }^{25}$ Finnish MAGIC Group: Finnish Centre for Astronomy with ESO, University of Turku, FI-20014 Turku, Finland ${ }^{26}$ Departament de Física, and CERES-IEEC, Universitat Autònoma de Barcelona, E-08193 Bellaterra, Spain 27 Japanese MAGIC Group: Physics Program, Graduate School of Advanced Science and Engineering, Hiroshima University, 739-8526 Hiroshima, Japan ${ }^{28}$ Armenian MAGIC Group: ICRANet-Armenia at NAS RA, 0019 Yerevan, Armenia ${ }^{29}$ Croatian MAGIC Group: University of Split, Faculty of Electrical Engineering, Mechanical Engineering and Naval Architecture (FESB), 21000 Split, Croatia ${ }^{30}$ Croatian MAGIC Group: Josip Juraj Strossmayer University of Osijek, Department of Physics, 31000 Osijek, Croatia ${ }^{31}$ Japanese MAGIC Group: Department of Physics, Kyoto University, 606-8502 Kyoto, Japan ${ }^{32}$ Japanese MAGIC Group: Department of Physics, Tokai University, Hiratsuka, 259-1292 Kanagawa, Japan ${ }^{33}$ Saha Institute of Nuclear Physics, HBNI, 1/AF Bidhannagar, Salt Lake, Sector-1, Kolkata 700064, India ${ }^{34}$ Inst. for Nucl. Research and Nucl. Energy, Bulgarian Academy of Sciences, BG-1784 Sofia, Bulgaria ${ }^{35}$ Japanese MAGIC Group: Department of Physics, Yamagata University, Yamagata 990-8560, Japan ${ }^{36}$ Finnish MAGIC Group: Astronomy Research Unit, University of Oulu, FI-90014 Oulu, Finland ${ }^{37}$ Croatian MAGIC Group: Ruđer Bošković Institute, 10000 Zagreb, Croatia ${ }^{38}$ INFN MAGIC Group: INFN Sezione di Perugia, I-06123 Perugia, Italy ${ }^{39}$ INFN MAGIC Group: INFN Roma Tor Vergata, I-00133 Roma, Italy ${ }^{40}$ Japanese MAGIC Group: Department of Physics, Konan University, Kobe, Hyogo 6588501, Japan ${ }^{41}$ also at International Center for Relativistic Astrophysics (ICRA), Rome, Italy ${ }^{42}$ now at Department for Physics and Technology, University of Bergen, NO-5020, Norway ${ }^{43}$ now at University of Innsbruck ${ }^{44}$ also at Port d'Informació Científica (PIC), E-08193 Bellaterra (Barcelona), Spain ${ }^{45}$ now at Ruhr-Universität Bochum, Fakultät für Physik und Astronomie, Astronomisches Institut (AIRUB), 44801 Bochum, Germany ${ }^{46}$ now at Department of Astronomy, University of California Berkeley, Berkeley CA 9472047 also at Dipartimento di Fisica, Università di Trieste, I-34127 Trieste, Italy 49 now at Laboratoire d'Annecy de Physique des Particules (LAPP), CNRS-IN2P3, 74941 Annecy Cedex, France ${ }^{50}$ also at INAF Trieste and Dept. of Physics and Astronomy, University of Bologna, Bologna, Italy 